\title{
Acute Cerebral Infarction Masked by a Brain Tumor
}

\author{
Kwo-Whei Lee ${ }^{a}$ Chung-Ping Lo ${ }^{b}$ \\ aDepartment of Radiology, Changhua Christian Hospital, Changhua, and \\ ${ }^{b}$ Department of Radiology, Buddhist Tzu Chi General Hospital, Taichung Branch, \\ Taichung, Taiwan, ROC
}

\section{Key Words}

Brain infarction - Brain neoplasms · Magnetic resonance imaging · Computed

tomography · Angiography

\section{Abstract}

We report on an 81-year-old man who presented with left limbs weakness and was brought to the emergency room where a brain computed tomography revealed a tumor at the right parasellar region. The patient was admitted to the neurosurgery department, and the symptoms were thought to be due to the tumor mass effect. The final diagnosis turned out to be acute ischemic infarction with an incidentally found brain tumor following angiography and magnetic resonance imaging.

\section{Introduction}

Computed tomography (CT) and magnetic resonance imaging (MRI) have been widely used for patients with acute neurological manifestations. However, these imaging techniques at times may be misleading, especially in a busy emergency room (ER) where doctors may have limited time to evaluate the patients thoroughly. The ability to differentiate between a brain tumor and ischemic infarction is sometimes a challenge, both clinically and radiologically. When they coexist, the clinician may fail to recognize that one is obscured by the other. Diffusion-weighted imaging (DWI) MRI provides better information for evaluating cases where the diagnosis is uncertain. Here, we report a case of acute cerebral infarction masked by a brain tumor on CT performed in the ER. DWI revealed concurrent cytotoxic edema due to acute ischemic infarction and vasogenic edema induced by the brain tumor. 


\section{Case Report}

An 81-year-old man who had been bed-ridden for a long period was brought to the ER after members of his family observed that he had had weakness of the left upper and lower limbs during the previous 2 days. A contrast-enhanced brain CT scan performed in the ER revealed a strongly-enhanced lesion over the right parasellar region of the middle cranial fossa with extensive perilesional brain edema (fig. 1a, b). The patient was then admitted to the neurosurgery department with the impression that he had a meningioma-induced mass effect associated with limb weakness. Two days later, he was referred by the neurosurgeon to the radiology department for preoperative transarterial tumor embolization. Right internal and external carotid angiography showed that the tumor was mainly supplied by the dural branches of the right internal carotid artery and was not supplied by the branches of the external carotid artery. We also noted that the right middle cerebral artery (MCA) was occluded at the M1 segment with absence of contrast opacification in this part of the artery (fig. 2a, b). We suspected that the first condition was an acute arterial thrombosis produced during the angiography. However, the patient did not develop any new neurological symptoms during the angiography procedure. After we had consulted with the neurologist and rechecked the clinical history provided by the patient's family members, we suspected that this finding was likely related to ischemic infarction prior to the admission, instead of arterial thrombosis during the angiography procedure. To prove this assumption, the patient was immediately sent for an MRI examination. Brain MRI showed an extra-axial tumor about $3 \mathrm{~cm}$ in size in the right parasellar region together with an acute ischemic infarction affecting the right cerebral hemisphere within the right MCA territory. DWI and the apparent diffusion coefficient (ADC) map revealed the coexistence of cytotoxic edema resulting from the acute ischemic infraction and vasogenic edema induced by the tumor (fig. $3 \mathrm{a}-\mathrm{d}$ ). The cytotoxic edema was extensive and was unlikely to be due to ischemic infarction produced during the angiography procedure within an interval of only $30 \mathrm{~min}$. The patient was referred to the neurology department for treatment of ischemic infarction and surgery was postponed.

\section{Discussion}

Brain tumor-induced arterial occlusion has been reported to be accompanied with both benign and malignant neoplasms, including meningioma, pituitary macroadenoma and malignant glioma $[1,2]$. The most common arterial occlusion usually occurs at the cavernous or supra-cavernous segment of the internal carotid artery due to encasement by a meningioma of parasellar location [1]. The mechanisms include gradual encasement or compression of the arterial walls that narrows the vascular lumen, and eventually thrombosis occurs [1]. The obstructive process is usually slow and progressive, which allows the collateral flow to develop. As a result, the patients may lack ischemic symptoms [1]. Aoki et al. [1] reported a patient presenting with acute onset of hemiplegia due to dissection of the MCA caused by invasion of a malignant glioma. In our case, the site of arterial occlusion was located at the right proximal MCA, which was not compressed or encased by the concurrent tumor in the parasellar skull base region. In addition, although the tumor had not been pathologically proven, based on imaging features it was more likely to be a benign lesion, such as a meningioma, rather than an invasive malignant neoplasm, which may invade or cause dissection of the arterial lumen [1]. We presumed that the ischemic infarction was not related to the tumor.

Many factors have been proposed regarding the development of meningioma-induced brain edema, including compression of the brain with venous stasis or brain ischemia, the effect of vascular endothelial growth factor, tumor secretory activity, and tumor invasion of the brain [2-7]. Tumor-induced brain edema is usually seen as a hypodense lesion with a 'finger-like' pattern distributed along the white matter on CT, whereas brain infarction usually involves both gray and white matter within a certain arterial territory. However, brain infarction may still be confused with or misdiagnosed as a brain tumor at times. In 
our case, the tumor-induced brain edema actually masked the ischemic infarction lesion which should have been evident on the CT scan during the 2nd day of clinical onset.

The diagnosis of cerebral infarction has been revolutionized by the widespread application of DWI, which is sensitive to alterations in the water molecular motion and allows the distinction between cytotoxic edema due to ischemic infarction and tumorinduced vasogenic edema [8]. Acute ischemic infarction shows areas of restricted water movement, which are hyperintense on DWI and hypointense on the ADC map. On the other hand, tumor-induced brain edema consists of free water movement in the interstitial space, which shows hypointensity on DWI and hyperintensity on the ADC map resembling cerebrospinal fluid. Besides DWI, perfusion-weighted imaging is another imaging sequence that can differentiate between the two disease entities by allowing assessment of cerebral blood flow, cerebral blood volume, and the presence (neoplasm) or lack (infarction) of neoangiogenesis [9].

Misdiagnosis of acute stroke is not uncommon, especially in the elderly and in demented patients. CT and MRI have improved the accuracy of diagnosis and the ability to differentiate acute stroke from other structural brain lesions, such as brain tumors and vascular lesions, and have had a great impact on patient treatment. With the development of functional imaging techniques such as DWI and perfusion-weighted imaging, diagnostic accuracy has been further improved. A meticulous clinical evaluation is always the key that leads to an accurate diagnosis, and doctors should not rely on only a single imaging modality to make a diagnosis in cases where the diagnose is uncertain. 

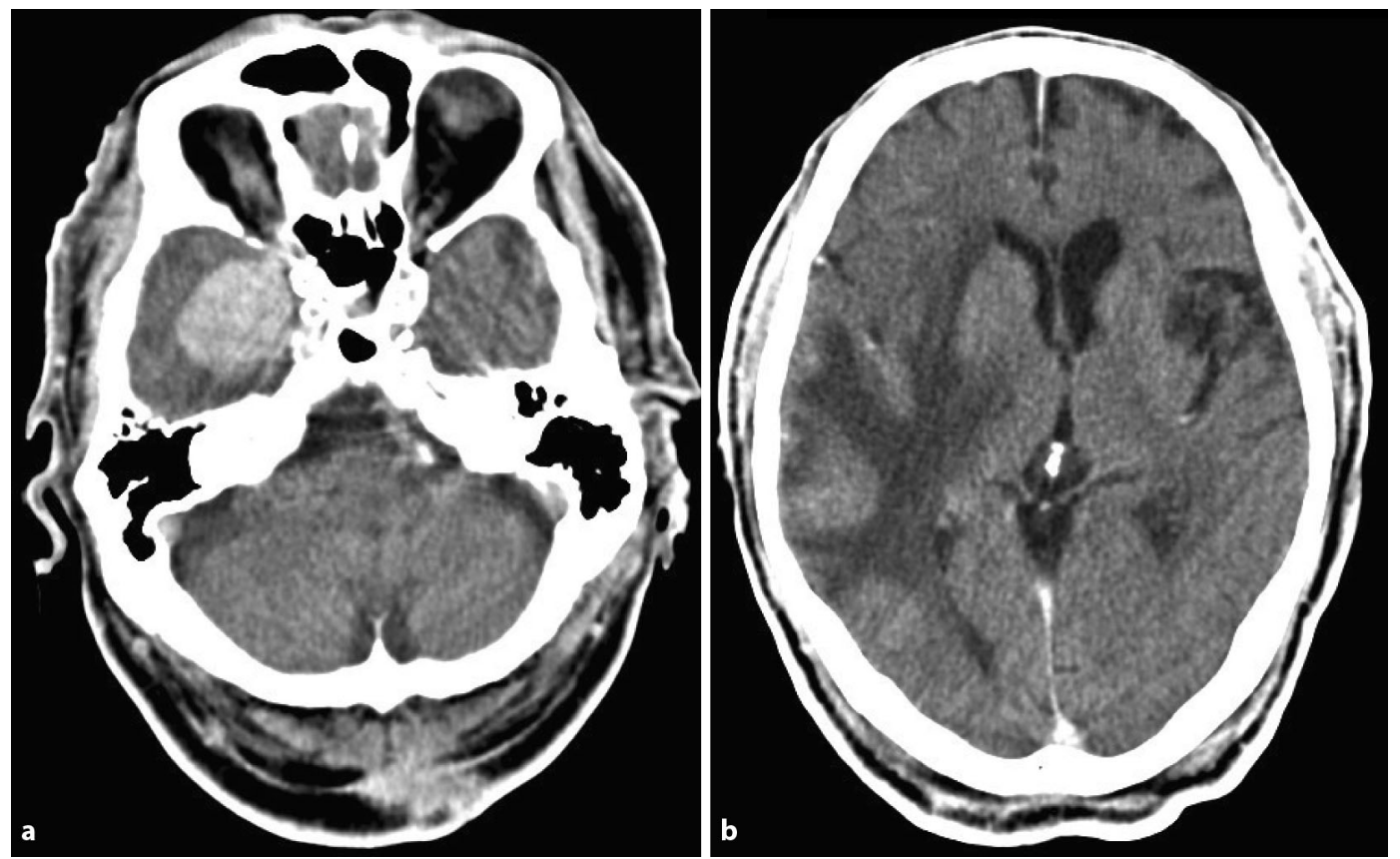

Fig. 1. a Axial contrast-enhanced brain CT scan reveals a right parasellar lesion. $\mathbf{b}$ There is a perilesional edema with a 'finger-like' pattern involving the right cerebral white matter. Ischemic infarction is obscured on this CT scan.
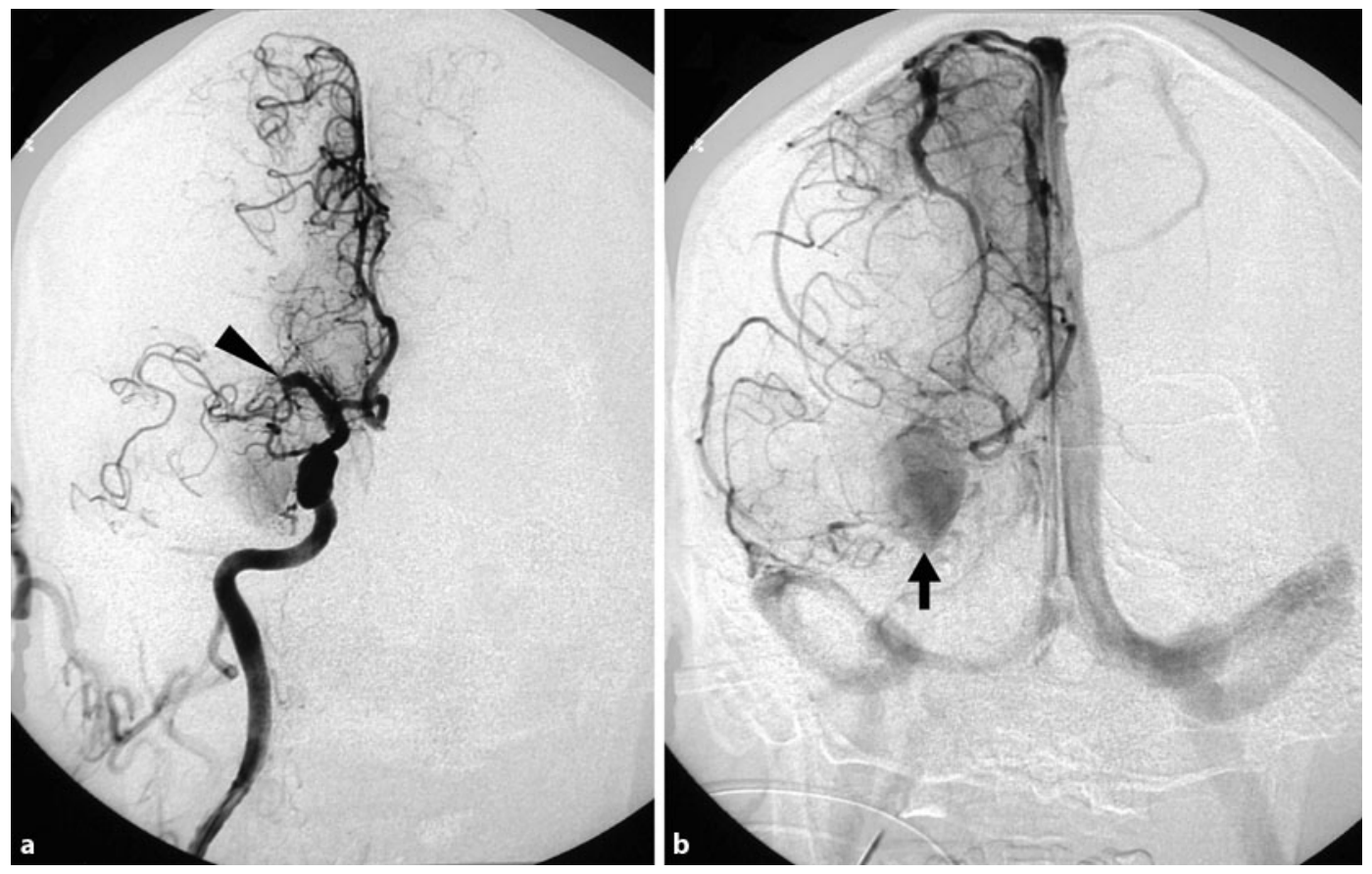

Fig. 2. a Anteroposterior projection of the right internal carotid angiography in the arterial phase demonstrates abrupt interruption of the right proximal MCA (arrowhead). b In the venous phase, the tumor stain was clearly seen (arrow). 

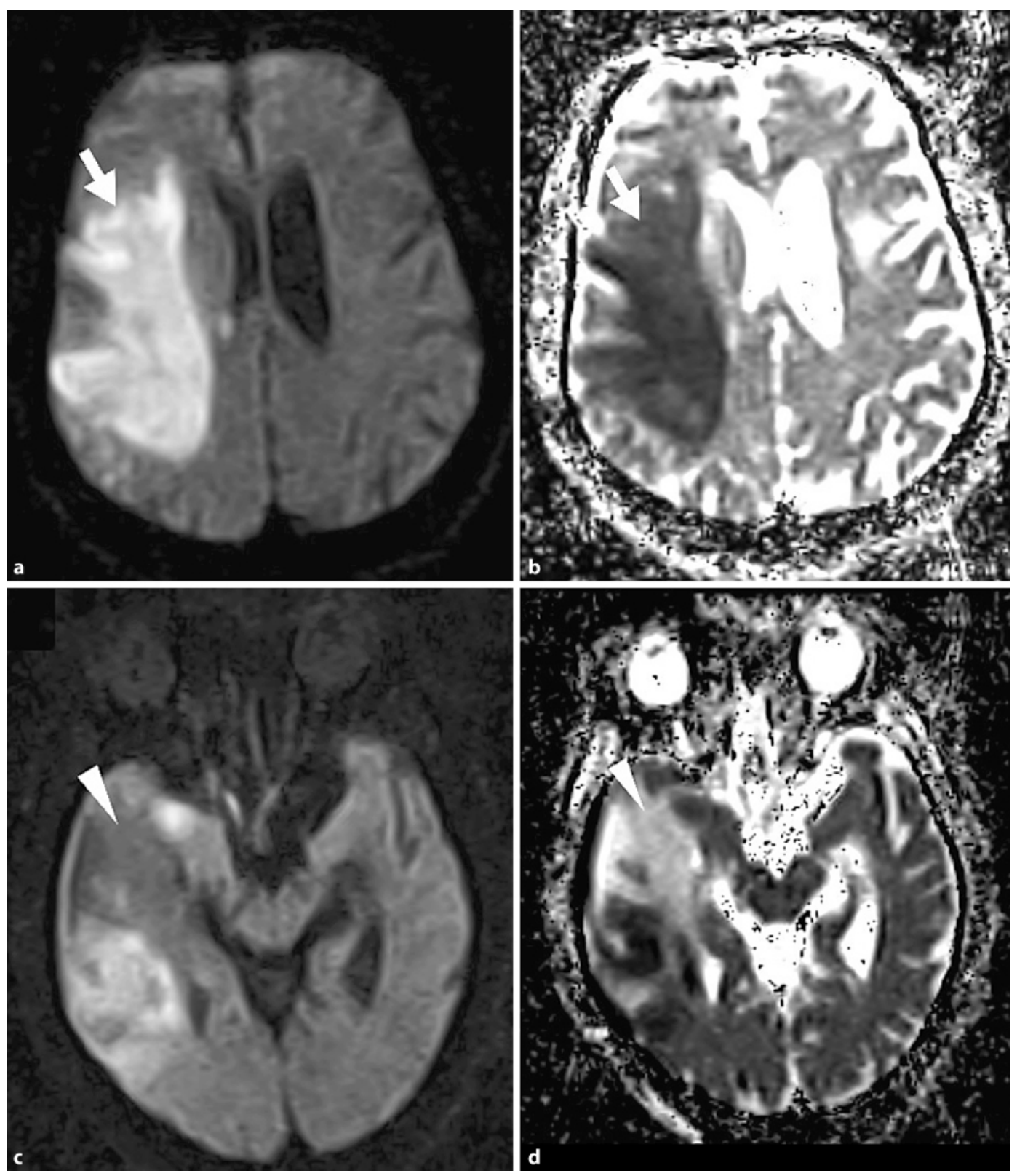

Fig. 3. DWI $\left(\mathrm{TR} / \mathrm{TE}=4521 / 110, \mathrm{~b}=1,000 \mathrm{~s} / \mathrm{mm}^{2} ; \mathbf{a}\right)$ and the corresponding ADC map $(\mathbf{b})$ show cytotoxic edema (arrows) resulting from the acute ischemic infarction. c, d At a lower cut, the tumorinduced vasogenic edema (arrowheads) is depicted. DWI clearly helps to differentiate between the two disease entities. 


\section{References}

1 Spallone A: Occlusion of the internal carotid artery by intracranial tumors. Surg Neurol 1980;15:51-57.

-2 Aoki N, Sakai T, Oikawa A, Takizawa T, Koike M: Dissection of the middle cerebral artery caused by invasion of malignant glioma presenting as acute onset of hemiplegia. Acta Neurochir (Wien) 1999;141:1005-1008.

-3 Bitzer M, Nägele T, Geist-Barth B, Klose U, Grönewäller E, Morqalla M, Heiss E, Voigt K: Role of hydrodynamic processes in the pathogenesis of peritumoral brain edema in meningiomas. J Neurosurg 2000;93:594-604.

4 Gurkanlar D, Er U, Sanli M, Özkan M, Sekerci Z: Peritumoral brain edema in intracranial meningiomas. J Clin Neurosci 2005;12:750-753.

5 Otsuka S, Tamiya T, Ono Y, Michiue H, Kurozumi K, Daiko S, Kambara H, Date I, Ohmoto T: The relationship between peritumoral brain edema and the expression of vascular endothelial growth factor and its receptors in intracranial meningiomas. J Neurooncol 2004;70:349-357.

6 Mattei TA, Mattei JA, Ramina R, Aguiar PH, Plese JP, Marino R Jr: Edema and malignancy in meningiomas. Clinics (Sao Paulo) 2005;60:201-206.

7 Uematsu H, Maeda M, Itoh H: Peritumoral brain edema in intracranial meningiomas evaluated by dynamic perfusion-weighted MR imaging: a preliminary study. Eur Radiol 2003;13:758-762.

-8 Domingo Z, Rowe G, Blamire AM, Cadoux-Hudson TA: Role of ischaemia in the genesis of oedema surrounding meningiomas assessed using magnetic resonance imaging and spectroscopy. Brit J Neurosurg 1998;12:414-418.

-9 Stadnik TW, Chaskis C, Michotte A, Shabana WM, van Rompaey KV, Luypaert R, Budinsky L, Jellus V, Osteaux M: Diffusion-weighted MR imaging of intracranial masses: comparison with conventional MR imaging and histologic findings. AJNR Am J Neuroradiol 2001;22:969-976.

10 Cha S, Knopp EA, Johnson G, Wetzel SG, Litt AW, Zagzag D: Intracranial mass lesions: dynamic contrastenhanced susceptibility-weighted echo-planar perfusion MR imaging. Radiology 2002;223:11-29. 